\title{
The effect of spatial frequency content on parameters of eye movements
}

\author{
Marina T. Groner • \\ Rudolf Groner • Adrian von Mühlenen
}

Received: 12 June 2007 / Accepted: 28 January 2008 / Published online: 15 October 2008

(C) Springer-Verlag 2008

\begin{abstract}
Two experiments were conducted to examine the influence of the spatial frequency content of natural images on saccadic size and fixation duration. In the first experiment 10 pictures of natural textures were low-pass filtered (0.04-0.76 cycles/deg) and high-pass filtered (1.9119.56 cycles/deg) and presented with the unfiltered originals in random order, each for $10 \mathrm{~s}$, to 18 participants, with the instruction to inspect them in order to find a suitable name. The participants' eye movements were recorded. It was found that low-pass filtered images resulted in larger saccadic amplitudes compared with high-pass filtered images. A second experiment was conducted with natural stimuli selected for different power spectra which supported the results outlined above. In general, low-spatial frequencies elicit larger saccades associated with shorter fixation durations whereas high-spatial frequencies elicit smaller saccades with longer fixation durations.
\end{abstract}

\section{Introduction}

In their pioneering research Buswell (1935) and Yarbus (1967) demonstrated that eye movements of observers looking at complex natural scenes depend on characteristics of the stimulus as well as on the intention of the observer.

M. T. Groner ( $₫)$

Department of Psychology,

University of Bern, CH 3000 Bern 9, Switzerland

e-mail: marina.groner@psy.unibe.ch

R. Groner

Institute for Research in Open-, Distance-, and eLearning,

Distance University of Switzerland, Brig, Switzerland

A. von Mühlenen

University of Warwick, Coventry, UK
Recently, the control of eye movements as part of an active vision system has become again an important topic not only for psychologists trying to understand the mechanisms of information-seeking but also for people designing artificial vision systems.

There is general consent that eye movements are guided by stimulus features as well as by cognitive processes. The question discussed at present is to what extent and under what conditions they are controlled bottom-up or top-down. One of the most important factors determining the pattern of eye movements is the nature of the task people are involved in. The locations of fixations in goal-directed actions are mainly controlled top-down, whereas in free viewing of a scene the influence of stimulus features can be demonstrated. Land and Hayhoe (2001) registered eye movements of people preparing food. Nearly all fixations were directed towards relevant objects before and during their handling. In a study by Turano, Geruschat, and Baker (2003) participants had to walk towards a target. A model using only bottom-up information could not explain their fixation behavior. Top-down information about the target's coarse features had to be included into the model.

There are studies that compared patterns of eye movements in different tasks. Henderson, Weeks, and Hollingworth (1999) let participants either search for a certain object in a scene or let them memorize the scene. They found a different pattern of fixations. When participants searched for an object they fixated it faster than when they had to memorize the scene. Henderson and Hollingworth (1998) compared eye movement parameters of scene viewing with those of reading. The variance and the mean of fixation duration and saccade length were greater in scene viewing than in reading. Similar results were found by Andrews and Coppola (1999) studying eye movements with different types of images and tasks. Viewing a 
complex natural scene and viewing simple textured patterns led to correlated eye movement parameters such as mean fixation duration and saccade length. The same result was found for reading and search but there was no correlation between the eye movement parameters of these tasks and those of viewing complex natural scenes and simple patterns. These studies demonstrate that free viewing of a scene leads to a different pattern of eye movements.

Under free viewing conditions, stimulus characteristics have been demonstrated to have an effect on fixation behavior. Deubel and Elsner (1986) found in a threshold perception task that saccades were larger when low spatial frequencies were viewed. In studies with above threshold perception, local features of the stimulus at fixation locations were compared with those features at randomly chosen or not-fixated locations. Several features such as edge density and contrast were found to be greater at the points of fixation (Mannan, Ruddock, \& Wooding, 1996, 1997; Parkhurst \& Niebur, 2003). In a similar study, Krieger, Rentschler, Hauske, Schill, and Zetzsche (2000) analysed second-order statistics of the stimuli and found that regions with higher spatial variance had a higher probability to be fixated.

The non-uniform distribution of features in an image can be modeled as saliency map and be used to predict fixation behavior in a pure bottom-up way. Itti and Koch (2000) implemented a computational model in which saliency was defined by intensity, color, and orientation. Parkhurst, Law, and Niebur (2002) applied this model to predict natural viewing of different types of images. They found that the pure bottom-up mechanisms of this model could contribute significantly to the explanation of fixation behavior. Tatler, Baddeley, and Gilchrist (2005) tested different saliency models to predict fixation locations in natural scenes. They found that contrast and edge information discriminated better between fixated and non-fixated locations, as compared to luminance and chromaticity, especially in high spatial frequency bands.

Most studies providing evidence for stimulus-driven, bottom-up control of eye movements are based on correlations between local stimulus features and the frequency with which these locations are being fixated. However, statistical correlation does not exclude the possibility that the local features attracting fixations are associated with informativeness about objects and therefore the control of eye movements runs top-down. To be able to interpret the results in a more causal way local features of the stimuli should be varied without much changing the informativeness about objects.

As a consequence of these considerations we varied the spatial frequency content of images and presented them to participants in a natural viewing task. Pictures of natural textures such as gravel, ice, bark, textiles, etc. were chosen as stimuli and digitally filtered, thus containing either the full range of spatial frequencies or only low-pass or highpass components. The cutoff frequencies were chosen according to the model of spatial channels by Wilson and Bergen (1979). We expect that, compared with unfiltered images, low-pass filtered stimuli will elicit larger saccadic sizes, while high-pass filtered images will elicit smaller saccades. Such a prediction can be related to studies which demonstrated something like a "saccadic responsive field size" as has been shown by double-step target studies (Becker \& Jürgens, 1979), multiple-target experiments (Menz \& Groner, 1986), or the center-of-gravity effect (Findlay, 1982) where it was demonstrated that the landing position of a next saccade is computed over an area of integration analogous to receptive field size.

With respect to fixation duration, two different hypotheses can be stated which contradict each other. The first hypothesis goes back to the well established result that low spatial frequency stimuli show shorter reaction times (Breitmeyer, 1975; Lupp, Hauske, \& Wolf, 1976) and shorter durations of visible persistence (Di Lollo \& Woods, 1981). These findings lead to the prediction that low-pass filtered images elicit shorter fixation durations. On the other hand, eye movement research in reading and other cognitive tasks usually show a positive correlation of fixation duration with saccadic size (Salthouse \& Ellis, 1980; Heller \& Müller, 1983). If saccadic size is larger with low spatial frequencies and smaller with high spatial frequencies, as stated above, then it should follow that low-pass filtered images will lead to longer fixation durations and high-pass filtered images to shorter fixations.

\section{Experiment 1}

Method

\section{Participants}

Eighteen undergraduate students (12 women and 6 men) participated in Experiment 1 in partial fulfilment of a course requirement. The average age was 27 years. Seven Participants had normal and 11 corrected-to-normal vision. Each participant served in a single session of about $30 \mathrm{~min}$.

\section{Apparatus}

The stimuli were presented on a 14" Eizo Flexscan 8060S monitor, which was driven by a PC through a Matrox graphics board (PIP-512/1024A). A head-chin rest helped participants in maintaining their head at the same position throughout a block of trials. Participants viewed the display monitor from a distance of $57 \mathrm{~cm}$. The total screen area subtended $20^{\circ} \times 27^{\circ}$ of visual angle. 
Eye movements were recorded with the OBER2 limbus tracker from Permobil Meditech. Eye position was sampled with a frequency of $200 \mathrm{~Hz}$ on both eyes. This recording system was driven by a second PC computer. The unprocessed signals were online plotted on the recording computer monitor, which was only visible to the experimenter. The eye movement data were stored on the recording computer's hard disc. The two computers were connected with each other by a parallel port cable to allow synchronisation between the display and the recording system and to set time markers in the data file for different events (calibration cross or stimulus onset) on the stimulus computer.

The laboratory was dimly illuminated to eliminate reflections on the CRT. The lamp was connected to a direct current converter to minimize interference with the eye movement recording.

\section{Stimuli}

All stimuli were based on ten photographs of natural textures taken from a standard work of textures. In a first step the photographs were digitized and saved in a computerized image format with a resolution of $512 \times 512$ pixels and 255 grey scale values. These digitized pictures were the base for all subsequent processing. Three different versions of stimuli textures were generated: In the first version the original images were used without being filtered. In the second version the images were low-pass filtered, including only frequencies within the range of $0.04-0.76$ cycles $/ \mathrm{deg}$. This range of spatial frequencies is assumed to be processed by the lowest spatial channel with a peak sensitivity of about 0.8 cycles/deg (Peterzell \& Teller, 1996). This channel corresponds to the U-channel in the model of Wilson and Bergen (1979). In the third version the images were high-pass filtered, including only frequencies within the range of 1.91$19.56 \mathrm{cycles} / \mathrm{deg}$. This range is assumed to be processed by the S- and N-channels in the Wilson and Bergen model. For some examples see Fig. 1. This produced a total of 30 stimuli textures, three versions (unfiltered, low-pass filtered, and high-pass filtered) for each of the ten texture images.

\section{Procedure}

The experiment was divided into one exercise- and ten testblocks. Each block started with a new calibration of the display-recording system. A cross was successively presented
Fig. 1 Three examples of stimuli in Experiment 1. The unfiltered images are presented in the left-hand column, the low-pass filtered images in the middle column, and the high-pass filtered images in the right-hand column
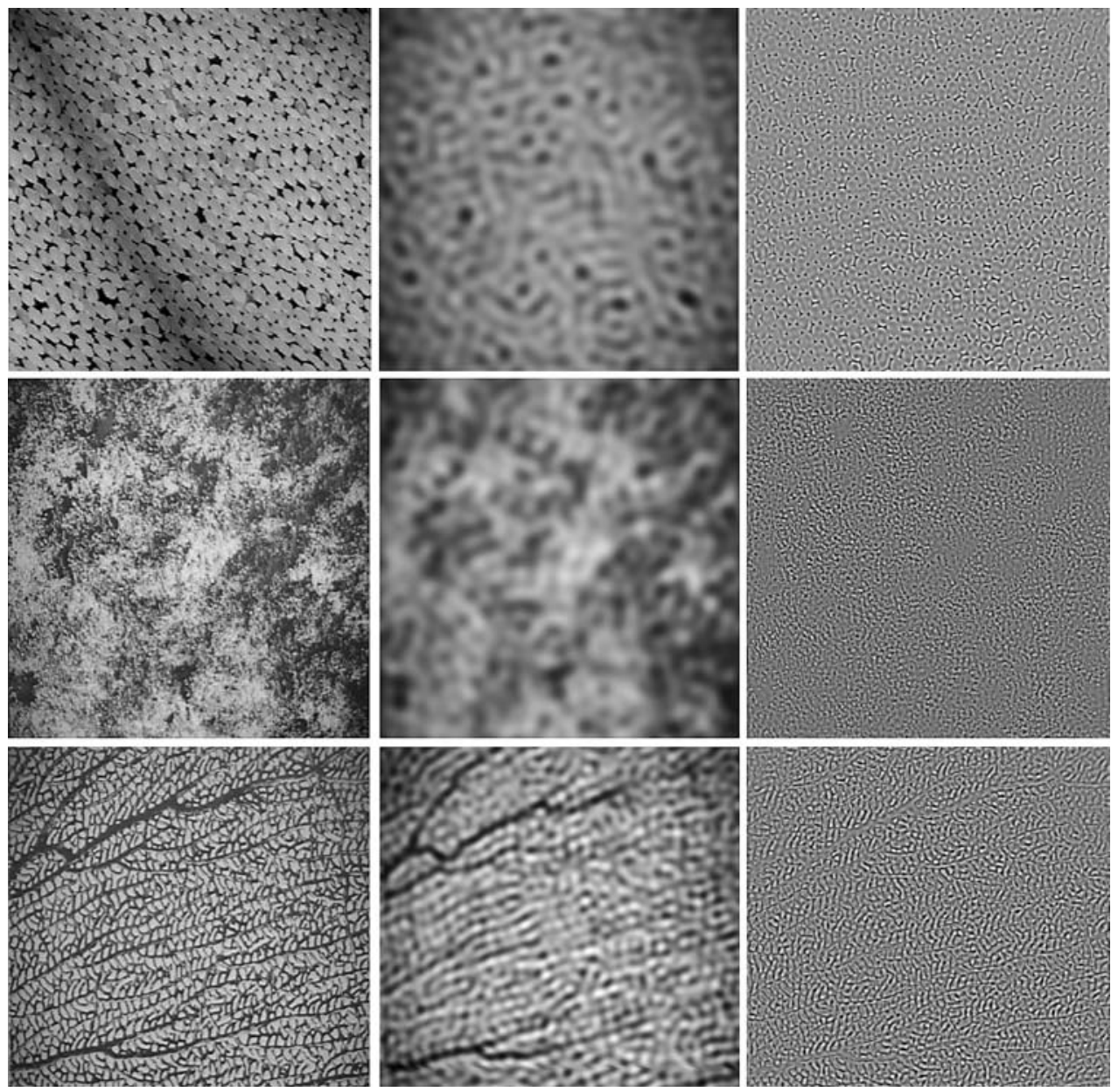
at 13 different positions on the screen (one at every position of a $4 \times 3$ matrix and twice at the screen centre). Each cross appeared for $1 \mathrm{~s}$ at its position, followed by a $1 \mathrm{~s}$ blank screen. Participants were told to fixate the cross while it was shown.

After calibration the three pictures of a block were shown. Each trial was started by the experimenter and it began with a central fixation cross which was on for $1 \mathrm{~s}$. Participants were told to fixate the cross, which was controlled by the Experimenter by looking at the approximate eye positions on the recording monitor. After the fixation cross disappeared the picture was presented for $10 \mathrm{~s}$.

Participants were asked to look at each picture for the whole $10 \mathrm{~s}$ it was presented. Their main task was to find a possible name for each of these pictures. After each picture there were $10 \mathrm{~s}$ to tell a name. If no name was given during this time, the experimenter suggested to call it "no title" and started the next trial. After each block there was a short break, where participants could remove their head from the head-chin rest.

\section{Design}

There were two variables: texture (1-10) and filter (unfiltered, low-pass, high-pass), giving a total of 30 pictures. The order of stimuli was for each participant randomly chosen, with the restraint that a block of consecutive pictures always contained an unfiltered, a low-pass filtered and a high-pass filtered image, which were generated from different textures. The order within a block was random.

\section{Data analysis}

The data were analysed using the software of WaltherMüller (1993). First the raw data were smoothed using a moving average (median) over three consecutive data points.

For calibration appropriate fixations were chosen for each cross shown on the screen (300 ms with least variation during stimulus onset). These values for the $\mathrm{x}$ - and $\mathrm{y}$-channel were used in a multiple linear regression analysis to estimate eye positions (least square) for the corresponding cross positions on the screen.

Saccadic eye movements and fixations were identified for both eyes separately with a noise adapting velocity threshold algorithm. The critical velocity was $36 \%$ s for saccade-begin and $18 \%$ s for saccade-end. Saccades that were smaller than $0.4^{\circ}$ of visual angle and fixations that were shorter than $40 \mathrm{~ms}$ were eliminated. The result of this automatic identification of fixations and saccades was plotted on a monitor and visually verified.

Based on visual inspection 4 participants had to be discarded from further analysis: For two of them some of the recordings were out of range. The other two produced a very noisy signal, which led to a bad fixation-saccade iden- tification. For all participants the same parameter settings were used.

Results

For each participant and each picture, fixation durations and saccadic size were averaged across the inspection interval of $10 \mathrm{~s}$. The resulting scores were subjected to analyses of variance with filtering and texture as factors with repeated measures. For saccadic size, there was a highly significant main effect of filtering, $F(2,26)=9.34, P<0.01$ ( $d f$ corrected by the Huynh-Feldt factor). For fixation duration, there was a significant effect of filtering, $F(2,26)=4.96, P<0.05(d f$ corrected by the Huynh-Feldt factor). The different textures had no effect on both variables, and no interaction was found.

Figure $2 \mathrm{a}$ shows the effects of filtering on the mean saccadic size. Low-pass filtered images and unfiltered images produced larger saccades than high-pass filtered images. The number of saccades being shorter than $3^{\circ}$ increased when high-pass filtered images were viewed (Fig. 2b). The effect of filtering on fixation durations is presented in Fig. 3. The mean duration of fixations was shorter with unfiltered images than with filtered images.

\section{Discussion}

The data from Experiment I showed that a change in the spatial frequency content of the images generated by filtering led to a change in the pattern of eye movements. Lowpass filtered images evoked larger saccades than high-pass filtered images. This seems plausible since low-pass filtered images have a coarser structure. The unfiltered images were close to the low-pass filtered images with respect to average saccadic size they evoked. This suggests that eye movements might be controlled by information from the lowest spatial frequency channel.

The images of textures used as stimuli did not have a significant effect on saccadic size or fixation durations, although their spatial frequency content varied. If our hypothesis holds then it should be possible to show such an effect by selecting images which vary more strongly in their power spectra than the ones used in the present experiment. This was realized in Experiment 2.

\section{Experiment 2}

Method

\section{Participants}

Twenty-four new participants (20 women and 4 men) took part in Experiment 2 in partial fulfilment of a course 

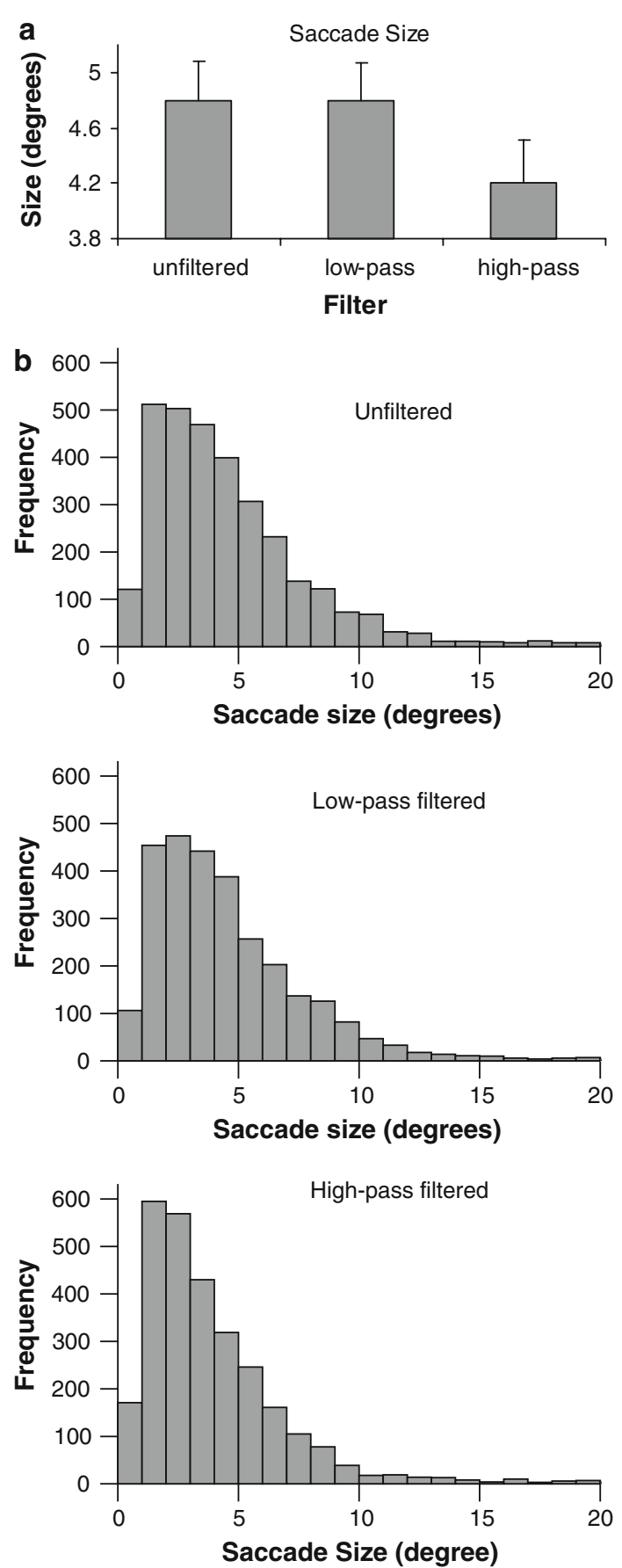

Fig. 2 a Mean saccadic amplitudes for the unfiltered, the low-pass filtered, and the high-pass filtered images. b Frequency distributions of saccadic amplitudes for the three types of images

requirement. The average age was 24 years. Twelve participants had normal and 12 corrected-to-normal vision. Each participant served in a single session of about $30 \mathrm{~min}$.

\section{Apparatus, procedure and stimuli}

Apparatus and procedure were the same as in Experiment 1. Again, ten stimuli textures were used as source material. In

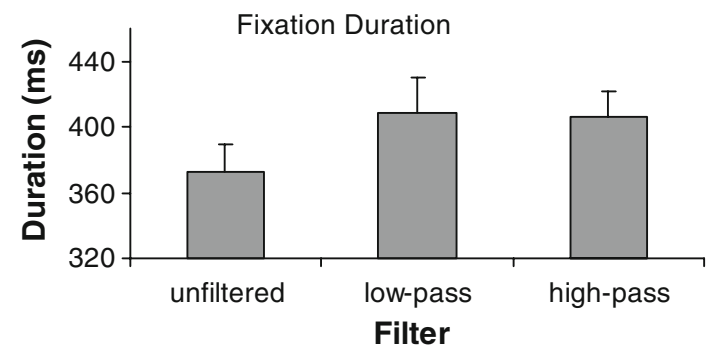

Fig. 3 Mean duration of fixation for the unfiltered, the low-pass filtered, and the high-pass filtered images

contrast to Experiment 1 they were chosen such that they varied in their power spectra. One group of textures called the "coarsies", was defined by high power in the low spatial frequency bands corresponding to the $\mathrm{U}$ - and $\mathrm{T}$ - channel in the model by Wilson and Bergen (1979). The other group of textures, called the "finies", was defined by high power in the high spatial frequency bands corresponding to the $\mathrm{S}$ - and N-channel in the model by Wilson and Bergen. For some examples of "finies" and "coarsies" see Fig. 4. Six textures were taken from the same collection of textures as in Experiment 1. One of the textures was the same as in Experiment 1, the others were new. A second difference concerned the filtering: Low-pass filtered pictures contained frequencies smaller than 2.5 cycles/deg, and highpass filtered pictures contained frequencies larger than 2.5 cycles/deg. The low-pass filtering contains the range of spatial frequencies assumed to be processed by the U- and T-channels in the model of Wilson and Bergen, the highpass filtering contains the range of spatial frequencies assumed to be processed by the S- and $\mathrm{N}$-channels in the same model.

\section{Design and data analysis}

There were three variables: texture (1-5), texture coarseness ("finies", "coarsies"), and filter (unfiltered, low-pass, high-pass), giving a total of 30 pictures. The order of stimuli was randomly chosen for each participant. The procedure for data analysis was the same as in Experiment 1. No participant had to be discarded.

\section{Results}

The eye movement data, saccadic size and fixation durations, were averaged for each participant and each group of textures across the inspection interval of $10 \mathrm{~s}$. The resulting scores were subjected to analyses of variance with filtering and spatial frequency content as factors with repeated measures. With respect to saccadic size, highly significant main effects of both factors, filtering, $F(2,46)=8.24, P<0.01$, ( $d f$ corrected by the Huynh-Feldt factor), and spatial 
Fig. 4 Four examples of stimuli in Experiment 2. In the first two rows are two examples of images with high power in low spatial frequencies, so-called "coarsies"; in the bottom two rows two examples of high power in high spatial frequencies, socalled "finies". The unfiltered images are presented on the left-hand column, the low-pass filtered images in the middle column, and the high-pass filtered images in the right-hand column
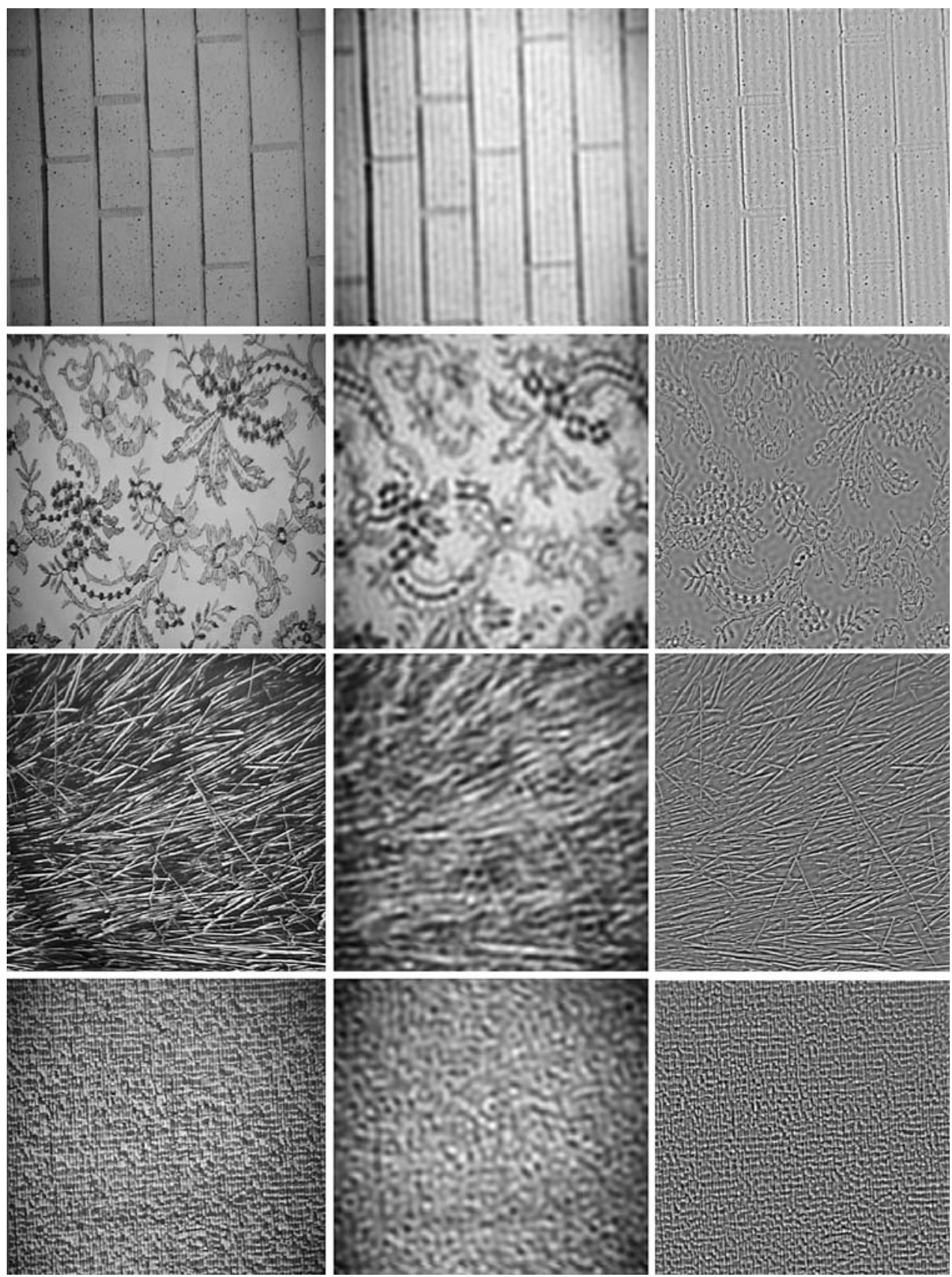

frequency content, $F(1,23)=9.82, P<0.01$, were found. There was no interaction between the two factors. With respect to fixation duration, a significant main effect of filtering, $F(2,46)=6.18, P<0.05$ ( $d f$ corrected by the Huynh-Feldt factor), and a highly significant main effect of spatial frequency content, $F(1,23)=62.92, P<0.001$, were found. Moreover there was a highly significant interaction between these two factors, $F(2,46)=8.89, P<0.01$ ( $d f$ corrected by the Huynh-Feldt factor).

Figure 5 shows the average size of saccades dependent on the type of filtering (unfiltered, low-pass filtered, and high-pass filtered), and on the spatial frequency content of the images. The images with high power in low-spatial frequencies, the "coarsies", elicited longer saccades than the "finies", the images with high power in high spatial frequencies. Filtering had the same effect in both groups of images. Low-pass filtering led to larger saccades than highpass filtering. This confirms the results of Experiment 1 . The mean saccadic size evoked by the unfiltered images lies in between.

In Fig. 6 the mean fixation durations are represented for the two experimental factors. Images with high power in high spatial frequencies, the "finies", evoked larger fixation durations than the "coarsies". Filtering had no effect when 


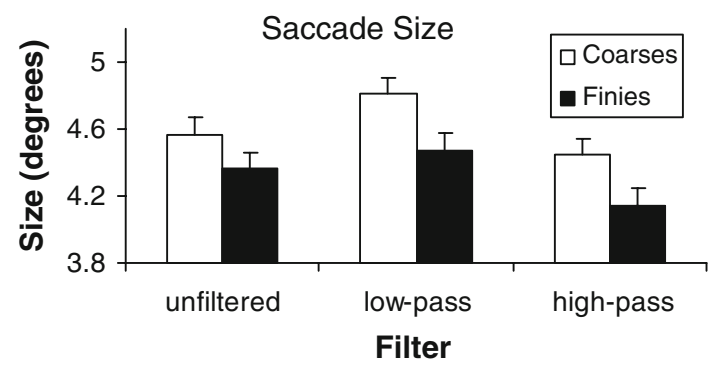

Fig. 5 Mean saccadic amplitudes for the unfiltered, the low-pass filtered, and the high-pass filtered images, separately for the "finies" and the "coarsies" textures

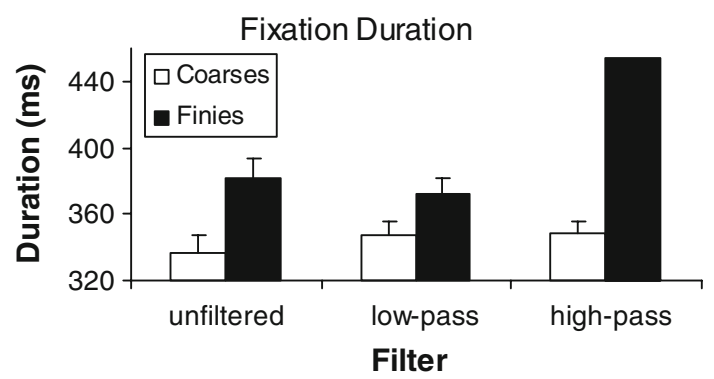

Fig. 6 Mean duration of fixation for the unfiltered, the low-pass filtered, and the high-pass filtered images, separately for the "finies" and the "coarsies" textures

the images were coarse. With the fine images, high-pass filtering produced longer fixations than no filtering or lowpass filtering. This partly replicates the results of Experiment 1 .

\section{General discussion}

Presumably three major factors play a role in the control of saccadic eye movements: perceptual knowledge about objects, strategies of information-seeking that are dependent on the task the visual system has to perform, and spatial features of the image. In our experiments we focused on the third factor and chose an experimental situation where the first two are supposed to be of minor importance. Both experiments in which participants inspected pictures of textures for $10 \mathrm{~s}$ in order to find a suitable name showed that characteristics of eye movements changed with the spatial frequency content of the images.

Low-pass filtered images increased the average saccadic sizes as compared to unfiltered or high-pass filtered images. The same result was found with respect to the spatial frequency content of unfiltered images. Images with high power in low-spatial frequencies led to larger saccades in the average than images with high power in high spatial frequencies. The spatial frequency content of the images showed also an effect on the average fixation duration. The longest mean fixation duration was found for high-pass filtered images with high power in high spatial frequencies, the shortest for unfiltered images with high power in low spatial frequencies. Mannan, Ruddock, and Wooding (1995) who had done similar experiments, found just the opposite: low-pass and high-pass filtered images evoked shorter saccades than unfiltered images, and fixation duration was longest with low-pass filtered images. One can only speculate about the reasons for the disagreement between these findings. The filtering of the images was different in both studies. However, this cannot account for the difference in results because we found the same effects in unfiltered images which just differed in spatial frequency content.

There was also a difference in the time that was available for participants to inspect the images, but again, it cannot explain the disagreement between the two studies because, essentially, we got the same results when we analysed just the first $3 \mathrm{~s}$ of our inspection intervals. It is more likely that the reason lies in the different tasks and material that led to different strategies for picking up information in the images. Participants in the study of Mannan et al. (1995) had to identify natural scenes with well-known objects whereas our participants had to find suitable titles for pictures of natural textures. A similar influence of stimulus material shows up in the study by Parkhurst et al. (2002). Stimulus features of fractal images made better predictions of fixation locations than stimulus features of natural scenes. The authors assume that the influence of top-down strategies is greater when scenes with well-known objects are observed.

The effect of different tasks has already been demonstrated in a study by Groner and Menz (1985). In a factorial experimental design, the influence of stimulus properties, tasks and individual differences between participants was investigated. With respect to saccadic size, there was a strong statistical interaction between tasks and participants. All participants showed shorter saccadic sizes in a visual search task, but with respect to the two other tasks (free inspection versus concept identification) the participants differed systematically from each other in their respective saccadic sizes.

The influence of the power spectra of the stimuli on eye movement parameters in our experiments suggests that the guidance of fixations was mainly stimulus-driven. High power in low spatial frequency bands led to greater saccade size. High power in high spatial frequency bands produced smaller saccade size. This effect was increased by taking bands of spatial frequencies out of the images. Given a certain fixation location, the planning of the next location depends on the information the visual system can get out of the peripheral parts of the visual field. The sensitivity for high spatial frequencies decreases with eccentricity. If there 
is high power in low spatial frequency bands or only low spatial frequencies are left in the image the visual system can analyse information further out in the peripheral visual field, thus enabling greater saccades to be made.

Acknowledgment We gratefully remember the many discussions with Dieter Heller in which he added his perspective, often resulting in a deeper understanding of the visual and cognitive processes under study. After his untimely death, we miss him strongly as a unique friend and colleague. This article is dedicated to his memory. This research was supported by grants of the Swiss National Science Foundation. Correspondence concerning this article should be addressed to: Marina Groner, Department of Psychology, University of Bern, CH-3000 Bern 9, Switzerland (e-mail: marina.groner@psy.unibe.ch).

\section{References}

Andrews, T. J., \& Coppola, D. M. (1999). Idiosyncratic characteristics of saccadic eye movements when viewing different visual environments. Vision Research, 39, 2947-2953.

Becker, W. R., \& Jürgens, R. (1979). An analysis of the saccadic eye movement system by means of double step stimuli. Vision Research, 19, 967-983.

Breitmeyer, B. G. (1975). Simple reaction time as a measure of the temporal response properties of the transient and sustained channels. Vision Research, 15, 1411-1412.

Buswell, G. (1935). How people look at pictures. A study of the psychology of perception in art. Chicago: University of Chicago Press.

Deubel, H., \& Elsner, T. (1986). Threshold perception and saccadic eye movements. Biological Cybernetics, 54, 351-358.

Di Lollo, V., \& Woods, E. (1981). Duration of visible persistence in relation to range of spatial frequencies. Journal of Experimental Psychology: Human Perception and Performance, 7, 754-769.

Findlay, J. M. (1982). Global processing for saccadic eye movements. Vision Research, 22, 1033-1045.

Groner, R., \& Menz C. (1985) The effects of stimulus characteristics, task requirements and individual differences on scanning patterns. In R. Groner, G. W. McConkie \& C. Menz (Eds.), Eye movements and human information processing (pp. 239-250). Amsterdam: North Holland.

Heller, D., \& Müller, H. (1983). On the relationship between saccadic size and fixation duration in reading. In R. Groner, C. Menz, D. F. Fisher \& R. A. Monty (Eds.), Eye movements and psychological functions: International views (pp. 287-302). Hillsdale: Lawrence Erlbaum.

Henderson, J. M., \& Hollingworth, A. (1998). Eye movements during scene viewing: An overview. In G. Underwood (Ed.), Eye guidance in reading and scene perception (pp. 269-293). Amsterdam: Elsevier.
Henderson, J. M., Weeks, P. A., Jr., \& Hollingworth, A. (1999). Effects of semantic consistency on eye movements during scene viewing. Journal of Experimental Psychology: Human Perception and Performance, 25, 210-228.

Itti, L., \& Koch, C. (2000). A saliency-based search mechanism for overt and covert shifts of visual attention. Vision Research, 40, 1489-1506.

Krieger, G., Rentschler, I., Hauske, G., Schill, K., \& Zetzsche, C. (2000). Object and scene analysis by saccadic eye-movements: An investigation with higher-order statistics. Spatial Vision, 13, 201-214.

Land, M. F., \& Hayhoe, M. (2001). In what ways do eye movements contribute to everyday activities? Vision Research, 41, 35593565 .

Lupp, U., Hauske, G., \& Wolf, W. (1976). Perceptual latencies to sinusoidal gratings. Vision Research, 16, 969-972.

Mannan, S., Ruddock, K. H., \& Wooding, D. S. (1995). Automatic control of saccadic eye-movements made in visual inspection of briefly presented 2-D images. Spatial Vision, 9, 363-385.

Mannan, S. K., Ruddock, K. H., \& Wooding, D. S. (1996). The relationship between the locations of spatial features and those of fixations made during visual examination of briefly presented images. Spatial Vision, 10, 165-188.

Mannan, S. K., Ruddock, K. H., \& Wooding, D. S. (1997). Fixation patterns made during brief examination of two-dimensional images. Perception, 26, 1059-1072.

Menz, C., \& Groner, R. (1986) Saccadic programming with multiple targets under different task conditions. In K. O'Regan \& A. LevySchoen (Eds.), Eye movements: From physiology to cognition (pp. 95-103). Amsterdam: Elsevier/North-Holland.

Parkhurst, D. J., \& Niebur, E. (2003). Scene content selected by active vision. Spatial Vision, 16, 125-154.

Parkhurst, D., Law, K., \& Niebur, E. (2002). Modeling the role of salience in the allocation of overt visual attention. Vision Research, $42,107-123$.

Peterzell, D. H., \& Teller, D. Y. (1996). Individual differences in contrast sensitivity functions: The lowest spatial frequency channels. Vision Research, 36, 3077-3085.

Salthouse, T. A., \& Ellis, C. L. (1980). Determinants of eye-fixation duration. American Journal of Psychology, 93, 207-234.

Tatler, B. W., Baddeley, R. J., \& Gilchrist, I. D. (2005). Visual correlates of fixation selection: Effects of scale and time. Vision Research, 45, 643-659.

Turano, K. A., Geruschat, D. R., \& Baker, F. H. (2003). Oculomotor strategies for the direction of gaze tested with a real-world activity. Vision Research, 43, 333-346.

Walther-Müller, P. U. (1993). Zur Messung von Augenbewegungen: Beschreibung der Software zur Analyse von Augenbewegungsdaten. Research Report 1993-3, Department of Psychology, University of Bern.

Wilson, H. R., \& Bergen, J. R. (1979). A four mechanism model for threshold spatial vision. Vision Research, 19, 19-32.

Yarbus, L. (1967). Eye movements and vision. New York: Plenum Press. 